\title{
Influence Of Sleeping In Hammocks On The Orthostatic Posture \\ ORIGINAL
}

\section{Abstract}

Background: Our research team believes that the arrangement of the hammock may influence the orthostatic posture. The aim of the present study was to assess the orthostatic posture in right and left views among hammock-users and hammock non-users of northeastern Brazil.

Methods: Were included 32 healthy participants, subdivided into two groups: hammock non-users, and hammock-users, both in the last 10 years. The orthostatic posture was performed through photogrammetry analysis (PAS/SAPO). We analyzed body alignment variables: horizontal alignment of the head, vertical alignment of the head, vertical alignment of the trunk, hip angle, body vertical alignments, horizontal alignment of the pelvis, knee angle, and ankle angle.

Results: On the right side, there was statistically significant difference in the horizontal alignment of the head $(P=0.04)$, hip angle $(P=0.02)$, vertical alignment of the body $(P=0.01)$ and horizontal alignment of the pelvis $(P=0.03)$. In the left side, there was statistically significant difference in horizontal alignment of the head $(P=0.04)$ and the horizontal alignment of the pelvis $(P=0.04)$.

Conclusion: Sleeping in the hammock influenced the orthostatic posture, especially in horizontal alignment of the head, hip angle, the vertical alignment of the body and horizontal alignment of the pelvis in the side views. Our data open new possibilities of research in this area, which has been obscure until this moment, and enable the indication or contraindication to sleep in hammocks.
Antonio de Pádua Rocha Nóbrega Neto1, Felipe Rodolfo Pereira da Silva², Any Carolina Cardoso Guimarães Vasconcelos ${ }^{3}$, Amélia Pasqual Marques ${ }^{4}$, Baldomero Antônio Kato da Silva ${ }^{5}$, Daniel Fernando Pereira Vasconcelos ${ }^{\text {* }}$

1 Master Student, Post Graduate Program in Biomedical Sciences, Federal University of Piaui, Parnaiba - PI, Brazil.

2 Biomedical Scientist, Federal University of Piaui, Parnaiba - PI, Brazil;

3 Assistant Professor, Department of Physical Therapy, Mauricio Nassau College, Parnaiba - PI, Brazil.

4 Associate Professor, Department of Physical Therapy, Speech Therapy and Occupational Therapy, Faculty of Medicine, University of São Paulo, São Paulo, Brazil.

5 Adjunct Professor; School of Physiotherapy, Federal University of Piaui, Parnaiba-PI, Brazil.

\section{Contact information:}

\section{Daniel Fernando Pereira Vasconcelos} Universidade Federal do Piauí - UFPI; Campus Ministro Reis Veloso; Av. São Sebastião, 2819, Reis Veloso; Parnaíba - PI - Brasil, 64204-035.

”vasconcelos@ufpi.edu.br

\section{Keywords}

Sleep system, Photogrammetry, alignment postural, Evaluation. 


\section{Background}

During the sleep, the musculoskeletal system may recover from the almost continuous loading of the everyday life, when the human body is in sustained contact with the sleep system (i.e., a mattress, supporting structure, and hammock). The hammock is commonly used by tropical areas as Brazil [1], and may provide a rocking with frequency similar to $0.25 \mathrm{~Hz}$, which accelerates sleep onset [2].

Our research team believes that the arrangement of the hammock may promote flexor position, and influence the angles and distances between body segments at a given point in time, the sleep posture. Such sleep posture can change the orthostatic posture, common standing position, via sensory systems such as: visual, somatosensory and vestibular [3]. Although no default approach to evaluate the posture has been defined, the photogrammetry has been used $[4,5,6,7]$. However, there is not a study that investigates the correlation between the hammock and posture.

The aim of the present study was to assess the orthostatic posture in right and left views among hammock-users and hammock non-users of northeastern Brazil.

\section{Method}

\section{Participants}

Were included 32 healthy participants, subdivided into two groups: Hammock-users (HA), in the last 10 years ( $n=15 /$ gender: 10 males and 5 female / age: $30.20 \pm 9.60$ / body mass index-BMl: $27.00 \pm$ 4.39) and Hammock Non-users ( $\mathrm{HN}$, in the last 10 years ( $n=17 /$ gender: 8 males and 9 female/ age: 25.94 \pm 5.83/ body mass index-BMI: 24.29 \pm 3.23). The study was conducted in the Federal University of Piaui, and has been approved by the Research Ethics Committee (0386.0.045.000-11/2011). All participants signed the free and informed term of consent.

The participants that presented some structural alteration, neurodegenerative or amputation and/ or shortening of the limbs, or no ancestry from northeastern Brazil were excluded.

\section{Photogrammetry through PAS/SAPO®}

The photogrammetry analysis was performed through the protocol of the Postural Assessment Software (PAS/SAPO) [5]. To summarize, for the achievement of the images, participants were individually evaluated, in the comfortable orthostatic posture, on a flat surface, barefoot, hair up and wearing bathing suit. For the calibration of the image in PAS/SAPO, a plumbline marked with two polystyrene balls $(15 \mathrm{~mm}$ diameter) at a distance of $100 \mathrm{~cm}$ from each other was used; black fabric 2.0x1.40 $\mathrm{m}$ for better visualization of the marked points; digital camera (Cannon, IXY Digital 700 model, 7.1 megapixels, 1.4x magnification); adjustable tripod for camera mount; double-sided tape to fix the polystyrene balls in the anatomical points (Figure 1).

We analyzed the participants in two views: right and left side. The following variables were analyzed (Figure 1): Horizontal alignment of the head - HHA; Vertical alignment of the head - HVA; Vertical alignment of the trunk - TVA; hip angle - HA; Vertical alignment of the body - BVA; Horizontal alignment of the pelvis - PHA; knee angle - KA; ankle angle - AA. After the images were captured in right side view, the carpet was rotated $180^{\circ}$ to obtain the photo in the left side view.

\section{Statistical Analysis}

For the statistical analysis we used Shapiro-Wilk to check the normal pattern of the data, and for parametric, and no parametric, t-student test and Mann-Whitney, respectively were used. (BioEstat ver.5.0, Belém, PA, Brazil). Significance level was established at $5 \%$. 
Figure 1: This figure shows the anatomic points, and variables analized throuh photogrammetry. 1) tragus, 2) spine process of $(7,3)$ acromion, 4) anterior-superior iliac spine, 5) posterior-superior iliac spine, 6) femur, greater trochanter, 7) knee, articular line, 8) lateral malleoli. The variables analyzed were: HHA - Horizontal alignment of the head, HVA - Vertical alignment of the head, TVA - Vertical alignment of the trunk, HA - Hip angle, BVA - Vertical alignment of the body, PHA - Horizontal alignment of the pelvis, KA - Knee angle, and AA - Ankle angle. Angles represented in red showed significant difference, and angles represented in blue denoted no statistical significance between hammock-users and hammock non-users.

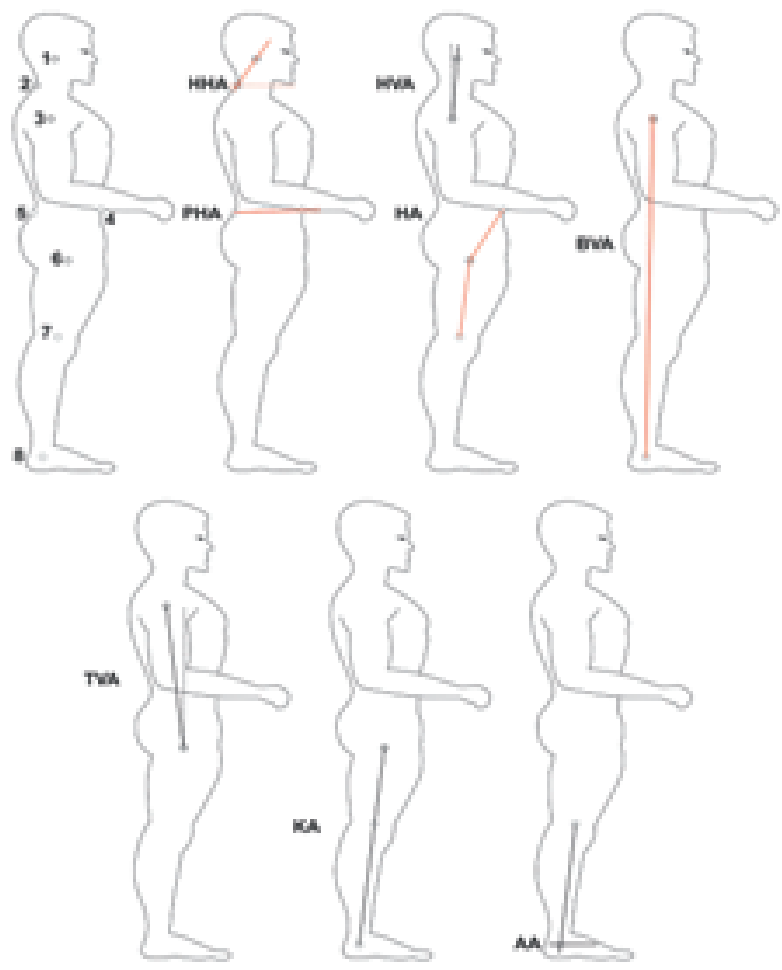

\section{Results}

On the right side, there was a statistically significant difference in horizontal alignment of the head $(P=0.04)$, hip angle $(P=0.02)$, vertical alignment of the body $(P=0.01)$, and the horizontal alignment of the pelvis $(P=0.03)$, as seen in table 1 .

On the left side, there was a statistically significant difference in the horizontal alignment of the head ( $P=0.04$ ) and horizontal alignment of the pelvis $(P=0.04)$ (Table 2$)$.

\section{Discussion}

This study is the first, according to our knowledge, that compared orthostatic posture among hammock-users and hammock non-users, and our data demonstrated that hammock-users influenced significantly the orthostatic posture, in the horizontal alignment of the head, hip angle, vertical alignment of the body, and horizontal alignment of the pelvis in the right side view, horizontal alignment of the head, and horizontal alignment of the pelvis 
Table 1. Variables analyzed through photogrammetry on the right side.

\begin{tabular}{|c|c|c|c|}
\hline \multirow{2}{*}{ Variables } & \multicolumn{2}{|c|}{ Mean \pm SD } & \multirow{2}{*}{ P value } \\
\cline { 2 - 4 } & HN & HA & $0.04^{*}$ \\
\hline Horizontal alignment of the head & $47.61 \pm 4.41$ & $43.93 \pm 5.68$ & 0.08 \\
\hline Vertical alignment of the head & $21.10 \pm 7.77$ & $16.47 \pm 6.98$ & 0.90 \\
\hline Vertical alignment of the trunk & $-1.57 \pm 2.26$ & $-1.66 \pm 1.98$ & $0.02^{*}$ \\
\hline Hip Angle & $-7.11 \pm 3.90$ & $-10.48 \pm 4.15$ & $0.01^{*}$ \\
\hline Vertical alignment of the body & $1.81 \pm 0.95$ & $2.62 \pm 0.78$ & $0.03^{*}$ \\
\hline Horizontal alignment of the pelvis & $-13.51 \pm 4.55$ & $-10.03 \pm 4.02$ & 0.06 \\
\hline Knee Angle & $-2.62 \pm 4.72$ & $-5.96 \pm 5.22$ & 0.94 \\
\hline
\end{tabular}

* indicates statistically significant difference. All variables were tested by t-student test. HN, Hammock Non-users; HA, Hammock-users.

Table 2. Variables analyzed through photogrammetry on the left side.

\begin{tabular}{|c|c|c|c|}
\hline \multirow{2}{*}{ Variables } & \multicolumn{2}{|c|}{ Mean \pm SD } & \multirow{2}{*}{$P$ value } \\
\hline & HN & HA & \\
\hline Horizontal alignment of the head $a$ & $47.50 \pm 3.27$ & $43.97 \pm 5.87$ & $0.04^{*}$ \\
\hline Vertical alignment of the head ${ }_{b}$ & $20.02 \pm 6.66$ & $18.64 \pm 7.98$ & 0.76 \\
\hline Vertical alignment of the trunk $b$ & $-1.08 \pm 1.99$ & $-1.18 \pm 1.85$ & 0.82 \\
\hline Hip Angle ${ }_{b}$ & $-8.89 \pm 3.62$ & $-10.83 \pm 6.25$ & 0.36 \\
\hline Vertical alignment of the body ${ }_{a}$ & $2.72 \pm 0.97$ & $2.94 \pm 1.06$ & 0.55 \\
\hline Horizontal alignment of the pelvis ${ }_{a}$ & $-13.75 \pm 4.25$ & $-10.71 \pm 4.25$ & $0.04^{*}$ \\
\hline Knee Angle ${ }_{b}$ & $-4.82 \pm 4.38$ & $-7.16 \pm 8.70$ & 0.38 \\
\hline Ankle Angle ${ }_{b}$ & $87.01 \pm 3.41$ & $87.50 \pm 4.13$ & 0.92 \\
\hline
\end{tabular}

* indicates statistically significant difference. a tested by t-student test. b tested by Mann-Whitney. HN, Hammock Non-users; HA, Hammock-users.

in left side view (Table 1 and 2). Age and BMI of all subjects were not significantly different between groups $(P>0.05)$. Such data suggest that the age and the BMI of individuals did not contribute with statistical difference observed in the angles.

Horizontal alignment of the head can indicate the position of the head relative to the trunk, when these values are decreased there is an indication that the head is inclined forwards [8]. The arrangement of the hammock in an arc promoted extended time for hammock-users in the same position, decreasing the angle of horizontal alignment of the head. However, the hammock non-users showed an increase in this angle, with their heads positioned posteriorly. The hammock arrangement does not stimulate changes in position and body movement. 
It seems even to distribute pressure on the bony prominences on the surface of the hammock, allowing the subject to remain longer in the same position, unlike a plane surface [9].

A study suggests that pain in the body may be associated with the sleeping position [10]. Our data open new possibilities regarding the indication and contraindication for the use of the hammock.

Therefore, its dynamics during sleep and the distribution of body pressure are different from other sleep systems.

Hip angle checks the position of the hip joint in relation to the flexion and extension. Body arrangement in the hammock may change the anterior and posterior muscular chains.

The vertical alignment of the body shows the anterior and posterior body inclination according to the vertical line. Even though our data showed significant differences in this angle, we similarly believe in the study [11] investigating that the mattress inflation pressure induced significant changes in the spinal alignment in normal subjects during sleep, but these values were of little physiological and clinical consequence. However, our data supply basic information necessary to evaluate similar correlations in other groups of individuals.

The horizontal alignment of the pelvis checks the angle of the pelvis, and is in a direct relation between the lumbar spine biomechanics, pelvis and hip [8]. In this way, hammock-users showed significantly decreased values for horizontal alignment of the pelvis, suggesting that it may compromise muscle groups and tissues corresponding to the contact surface.

The exact role of the hammock in the orthostatic posture is not yet clear. According to our data, it is acceptable that the hammock may influence on the orthostatic posture, because this sleep system is different in the following aspects: the rocking, the arrangement, the pressure over the bony prominences, the body movements and change positions during the sleep, and others that still remain ob- scure. Our research team demonstrated that the arrangement of the hammock may promote flexor position, and influence the angles and distances between body segments.

\section{Conclusion}

Sleeping in the hammock influenced the orthostatic posture, especially in the horizontal alignment of the head, hip angle, vertical alignment of the body and horizontal alignment of the pelvis in the side views. Our data open new the possibilities of research in this area, and enable the indication or contraindication for the sleep in hammocks.

\section{Competing interest}

We declare no conflict of interest.

\section{Author's contribution}

APRNN, ACCGV and FPRS participated in the acquisition of data and wrote the manuscript. APRNN interpreted the data and drafted the manuscript. DFPV, APM and ACCGV determined the design and drafted the manuscript. BAKS, DFPV and APM review of the manuscript. All authors read and gave final approval for the version submitted for publication.

\section{Acknowledgments}

Research supported by the Federal University of Piaui (UFPI - Edital PIBIC 2013/2014 and BIAMA 03/2014). We thank teacher Abilio Borghi for the grammar review of the manuscript. 


\section{References}

1. Haex B. Back and bed: ergonomics aspects of sleeping. Boca Raton London New York Washington, D.C.: CRC PRESS 2004, p. 35-69.

2. Bayer L, Constantinescu I, Perrig S, Vienne J, Vidal PP, Mühlethaler M, Schwartz S: Rocking synchronizes brain waves during a short nap. Curr Biol 2011, 21(12):R461-2.

3. Jacobs JV, Horak FB. Cortical control of postural responses. J Neural Transm 2007, 114(10):1339-48

4. Iunes DH, Castro FA, Salgado HS, Moura IC, Oliveira AS, Bevilaqua-Grossi D: Confiabilidade intra e interexaminadores e repetibilidade da avaliação postural pela fotogrametria. Rev Bras Fisioter 2005, 9(2):327-34

5. Ferreira EAG, Duarte $M$, Maldonado EP, Burke TN, Marques AP: Postural assessment software (PAS/SAPO): Validation and reliabiliy. Clinics 2010, 65(7):675-681.

6. Lopes AJ, da Silva DP, Kasuki L, Gadelha MR, Camilo GB, Guimarães FS: Posture and balance control in patients with acromegaly: results of a cross-sectional study. Gait Posture 2014, 40(1):154-9.

7. Ferreira EA, Duarte $M$, Maldonado EP, Bersanetti AA, Marques AP: Quantitative assessment of postural alignment in young adults based on photographs of anterior, posterior, and lateral views. J Manipulative Physiol Ther 2011, 34(6):371-380.

8. Krawczky B, Pacheco AG, Mainenti MRM: A Systematic Review of the Angular Values Obtained by Computerized Photogrammetry in Sagittal Plane: A Proposal for Reference Values. J Manipulative Physiol Ther 2014, 37(4):269-275.

9. Werner CM, Ossendorf C, Meyer DC, Blumenthal S, Gerber C: Subacromial pressures vary with simulated sleep positions. J Shoulder Elbow Surg 2010, 19(7):989-93.

10. Zenian J: Sleep position and shoulder pain. Med Hypotheses 2010, 74(4):639-643.

11. Lahm R, laizzo PA: Physiologic responses during rest on a sleep system at varied degrees of firmness in a normal population. Ergonomics 2002, 45(11):798-815.

\section{Comment on this article:}
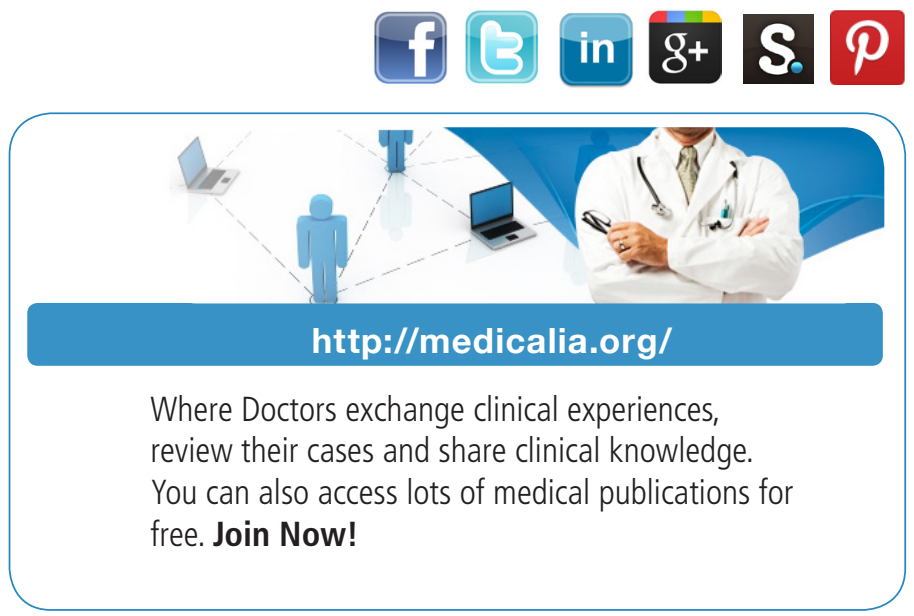

\section{Publish with iMedPub}

\section{http://www.imed.pub}

International Archives of Medicine is an open access journal publishing articles encompassing all aspects of medical science and clinical practice. IAM is considered a megajournal with independent sections on all areas of medicine. IAM is a really international journal with authors and board members from all around the world. The journal is widely indexed and classified Q1 in category Medicine. 